\title{
Not so self-centred after all
}

DOI:

10.1038/nri2202

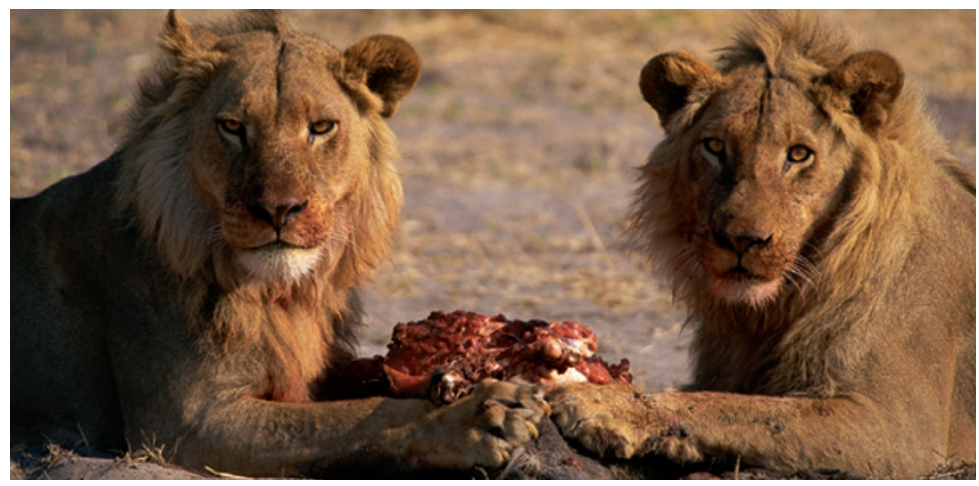

Contrary to popular belief, a new study suggests that forkhead box P3 (FOXP3)-expressing regulatory T cells preferentially recognize non-self antigens rather than self antigens. This study, published in Immunity, questions the theory that some thymocytes that recognize selfpeptide-MHC complexes with high affinity, instead of dying by negative selection, acquire FOXP3 expression and differentiate into regulatory $\mathrm{T}$ cells with specificities that mirror autoreactive T cells. Pacholczyk et al. compared hundreds of T-cell receptor (TCR) sequences and antigenic specificities from regulatory $\mathrm{T}$ cells and naive $T$ cells and found little evidence for self-reactive regulatory $\mathrm{T}$ cells.

To compare TCR diversity on a manageable scale, Pacholczyk et al. analysed T cells from mice expressing a variable TCR $V \alpha$ chain and a fixed transgenic $V \beta$ chain; this limits the repertoire but preserves a certain degree of diversity. Their analysis showed that about $70 \%$ of the most frequent TCRs on naive $\mathrm{T}$ cells were also found on regulatory $\mathrm{T}$ cells, although the frequency of any particular TCR varied between the subsets. This surprisingly high degree of overlap and varying frequency suggests that these two subsets arise from asymmetric distribution of the same TCRs, and not separate selection of different TCRs.

Based on the idea that the expression of high-affinity TCRs improves a T cell's ability to homeostatically expand, the authors compared the levels of in vivo homeostatic expansion by naive and regulatory $\mathrm{T}$ cells. They found no differences - cells from both subsets expanded at similar rates, regardless of whether they expressed TCRs that were shared or not shared.

A major obstacle to the determination of antigenic specificity of regulatory $\mathrm{T}$ cells has been their inherent anergic phenotype.

However, Pacholczyk et al. got around this by generating T-cell hybridomas bearing TCRs from regulatory $\mathrm{T}$ cells. Although the resultant hybridomas lost FOXP3 expression, they acquired the ability to secrete interleukin-2 on TCR stimulation, thereby facilitating the assessment of TCR reactivity. The authors also used mice in which the restricted TCR repertoire has been raised against a single peptide-MHC complex ( $\mathrm{A}^{\mathrm{b}} \mathrm{Ep}$; considered self $)$ and therefore is not tolerant to natural, endogenously processed peptides (considered non-self). A substantial proportion of hydridomas expressing
TCRs derived from either regulatory $\mathrm{T}$ cells or naive $\mathrm{T}$ cells from these mice showed reactivity to splenocytes presenting endogenous peptides (non-self), whereas none reacted with splenocytes presenting $\mathrm{A}^{\mathrm{b}} \mathrm{Ep}$ (self) complexes. This was not because the ubiquitous expression of a single peptide-MHC complex caused the deletion of self-reactive $\mathrm{T}$ cells, as hybridomas generated from $\mathrm{T}$ cells (both regulatory or naive) that were selected on a normal array of peptide-MHC complexes also showed no self reactivity. These observations support the concept that the TCR repertoires of both T-cell subsets are comparable, being biased towards non-self rather than self peptides.

Finally, the authors showed that colitis could only be induced by adoptive transfer of naive $\mathrm{T}$ cells to lymphopenic mice if the recipient mice expressed wild-type peptide-MHC-class-II complexes but not if they expressed a single peptide-MHC-class-II complex. This indicates that reactivity to non-self antigens rather than to self antigens is responsible for disease in this model.

So, given that these authors found little evidence of self-reactive regulatory $\mathrm{T}$ cells, they propose that, similar to conventional T cells, the selecting self peptide for regulatory $\mathrm{T}$-cell differentiation is different from the peptide that activates the regulatory $\mathrm{T}$ cells in the periphery.

Lucy Bird

ORIGINAL RESEARCH PAPER Pacholczyk, R. et a Nonself-antigens are the cognate specificities of Foxp3+ regulatory T cells. Immunity 27, 493-504 (2007)

FURTHER READING Stephens, G. L. \& Shevach, E. M. Foxp $3^{+}$regulatory T cells: selfishness under scrutiny. Immunity 27, 417-419 (2007) 